\title{
Klinikärzte - mehr Arbeit in kürzerer Zeit! Chirurgen haben die höchste Arbeitsbelastung
}

Wenn man hört, dass die Wochenarbeitszeit der Kliniker in den letzten zehn Jahren im Schnitt um fünf Stunden gesunken ist, klingt das zunächst erfreulich. Die tatsächliche Belastung der Krankenhausärzte ist allerdings eher weiter gestiegen. Schuld daran sind die stetige Verdichtung der Arbeit, der höhere bürokratische Aufwand und die steigenden Fallzahlen. Dass dabei gesundheitliche Folgen nicht ausbleiben, wird keinen wundern. In Hamburg beispielsweise schwebt bereits jeder Dritte Krankenhausarzt in Gefahr, ein Burnout-Syndrom zu erleiden.

Immerhin: Die Arbeitsbelastung, der sich Ärzte und vor allem auch Krankenhausärzte heute gegenüber sehen, wird von den Deutschen durchaus gewürdigt. Einer repräsentativen Umfrage (1) zufolge sagten immerhin $87 \%$ der Befragten, Krankenhausärzte hätten einen anstrengenden Beruf, könnten sich für den einzelnen Patienten nur wenig Zeit nehmen $(71 \%)$ und haben viel mit Bürokratie zu kämpfen (64\%).

Das klingt so, als ob sich nach dem Urteil des Europäischen Gerichtshofs vor einigen Jahren an der Situation der Ärzte überhaupt nichts geändert habe. Tatsächlich hat sich in den letzten zehn Jahren - zumindest in Hamburg, wie eine aktuelle Analyse vom Team um den Arbeitsmediziner Dr. Ralf Wegener dokumentiert - die Arbeitszeitbelastung der Ärzte quantitativ eher verringert (3).

\section{Trotz geringfügig gesunkener} Arbeitszeiten: keine Entwarnung In Hamburg sank die mittlere wöchentliche Arbeitszeit im Mittel um knapp fünf Stunden pro Woche auf 56,6 Stunden. Dies gilt vor allem für die Innere Medizin: Hatten die Internisten der Hamburger Kliniken 1997 im Schnitt noch 64 Stunden gearbeitet, leisten sie heute „nur“ noch 55 Stunden Dienst pro Woche. Verbessert hat sich auch die Situation in der Chirurgie, wobei hier allerdings noch immer rund 60 Wochenarbeitsstunden die Regel sind - ähnlich übrigens wie auch in der Pädiatrie oder der Gynäkologie.

Profitiert haben von der Arbeitszeitverkürzung vor allem die Assistenzärzte (62,7 versus 56,1 Stunden), Chef- und vor allem Oberärzte haben dagegen daran nur einen geringen Anteil. Das heißt aber nicht, dass Assistenzärzte beruflichen Belastungen weniger stark ausgesetzt sind - eher im Gegenteil. Betrachtet man den prozentualen Anteil der deutlich erhöhten Arbeitsbelastung liegen sie mit 21,2\% deutlich vor den Chef- und Oberärzten mit $13,7 \%$. Am stärksten getroffen sind dabei operativ tätige Ärzte, Jüngere (bis 35 Jahre) und Männer (2). dieser Zahlen also keine Rede sein. Nur 15 \% der vollzeitbeschäftigten Ärzte in Deutschland geben an, nicht mehr als neun Stunden pro Arbeitstag abzuleisten, was der üblichen Vollzeitbeschäftigung von 38,5-42 Wochenstunden entspricht. Fast 23 \% allerdings arbeiten an einem durchschnittlichen Werktag sogar mindestens elf Stunden (2).

\section{Arbeit verdichtet sich immer weiter}

Dazu kommt noch ein weiteres gravierendes Problem, denn die Arbeit der Krankenhausärzte hat sich in den letzten Jahren kontinuierlich verdichtet: Der Zeitaufwand für administrative Tätigkeiten erhöht sich von Jahr zu Jahr, die Liegezeiten werden kürzer, die Fallzahlen steigen. Insgesamt führt dies zu einer höheren Beanspruchung der Kliniker. Spitzenreiter in der Liste der besonders belasteten Ärzte sind die Chirurgen, erst mit einigem Abstand folgen Gynäkologen, Urologen und die Internisten. Vergleichsweise ruhig zu geht es in der Anästhesiologie, Radiologie und Psychiatrie bzw. Psychotherapie.
Von einer Entwarnung kann anhand
Die Folge: Geringere Qualität, höhere Risiken, Burnout Dass fast jeder, der beruflich stark belastet ist, dafür einen Preis zahlen muss, ist wohl unbestritten. Über die Einschnitte in der Lebensqualität wird man nicht lange diskutieren. Auch dass überlange Arbeitszeiten die Qualität der Leistung mindern oder das Risiko sich selbst zu verletzen und die Unfallgefahr erhöhen ist zweifellos richtig. Die Arbeitszeitbelastung gilt aber auch als Hauptursache von Distress, ein Auslöser für bestimmte mentale und somatische Krankheiten, wie unter anderem das Burnout-Syndrom.

Arbeitsbedingungen im Ausland
sind mal besser, mal schlechter
Die Arbeitszeitbelastung der Krankenhaus-
ärzte ist übrigens kein isoliertes deutsches
Problem. In England beträgt die Arbeitszeit
in den Kliniken im Schnitt ebenfalls 60 Stun-
den - allerdings bei deutlich besserer Bezah-
lung. In den Vereinigten Staaten sind sogar
Wochenarbeitszeiten von 85 Stunden keine
Seltenheit. Norwegen, Neuseeland, Öster-
reich und die Schweiz dagegen bieten den
Ärzten bessere Arbeitsbedingungen. Da mag
man sich nicht mehr wundern, wenn immer
mehr Ärzte ins Ausland „abwandern“.

Laut der Hamburger Ärztebefragung hat sich der Anteil der vom Burnout gefährdeten Mediziner in den letzten zehn Jahren mehr als verdoppelt und liegt jetzt um die $30 \%$. Besonders betroffen sind die chirurgisch tätigen Chef- und Oberärzte, allerdings nicht in den Universitätskliniken, wie es noch 1997 der Fall war und man vielleicht auch heute eher vermuten würde, sondern in den Allgemeinen Krankenhäusern. Stephanie Schikora, Stuttgart

\footnotetext{
Literatur

1 MLP Gesundheitsreport 2007

2 Rosta I. Deutsches Ärzteblatt 2007; 104 (36): A2417-A2423

3 Wegner R, et al. Hamburger Ärzteblatt 2007; (11): 515-518
} 